\title{
Caracteristicas fitoquímicas y capacidad antioxidante in vitro de Aloe vera, Plukenetia volubilis, Caiophora carduifolia, Cecropia membranácea
}

\author{
In vitro phytochemical characteristics and antioxidant capacity of \\ Aloe vera, Plukenetia volubilis, Caiophora carduifolia, Cecropia \\ membranacea
}

\author{
César Franco-Quino ${ }^{1,2}$, Danny Muñoz-Espinoza ${ }^{1,3}$, César Gómez-Herreros ${ }^{1}$, Gina \\ Chau-Miranda' ${ }^{1}$ Liliana Cueva-Piña ${ }^{1}$, Eliza Guardia-Ortiz' ${ }^{1}$ Santos Saavedra-Yucra', \\ Jorge Arroyo-Acevedo ${ }^{4,5}$, Oscar Herrera-Calderón ${ }^{6}$ \\ 1 Unidad de Posgrado, Facultad de Farmacia y Bioquímica, Universidad Nacional Mayor de San Marcos, Lima, Perú. \\ ${ }^{2}$ Laboratorio de Fisiología y Farmacología, Facultad de Odontología, UNMSM, Lima, Perú. \\ ${ }^{3}$ Facultad de Ciencias Quimicas, Universidad de Guayaquil, Ecuador. \\ ${ }^{4}$ Laboratorio de Farmacología Experimental, Facultad de Medicina Humana, UNMSM, Lima, Perú. \\ ${ }^{5}$ Instituto de Investigaciones Clínicas, UNMSM, Lima, Perú. \\ ${ }^{6}$ Facultad de Fisiología y Fisiopatología, Facultad de Farmacia y Bioquímica, Universidad Nacional San Luis Gonzaga, Ica, Perú.
}

\begin{abstract}
Resumen
Introducción. Los antioxidantes han demostrado potencial quimioprotector en patologías degenerativas, inflamatorias, autoinmunes, oncológicas y asociadas al distrés respiratorio. Objetivo. Evaluar las características fitoquímicas y capacidad antioxidante in vitro mediante el método DPPH y ABTS. Diseño. Observacional analítico. Lugar. Laboratorio de Farmacología Experimental, Facultad de Medicina Humana, Universidad Nacional Mayor de San Marcos, Lima, Perú. Material biológico/Químico. Hojas de Aloe vera, semillas de Plukenetia volubilis, hojas-tallos de Caiophora carduifolia, hojas de Cecropia membranacea. Intervenciones. Observación y análisis de la capacidad antioxidante mediante el método DPPH-concentración efectiva media $\left(\mathrm{CE}_{50}\right)$ de los extractos y la capacidad antioxidante equivalente a trolox por el método del ABTS. Medida de resultados. Marcha fitoquímica preliminar, porcentaje de inhibición antioxidante por captación del radical DPPH, determinación del equivalente trolox/g $g_{\text {extracto }}$ Resultados. La Cecropia membranacea presentó mayor número de metabolitos secundarios, alcaloides, saponinas, compuestos flavonoides; en la captación de radicales $\mathrm{DPPH}$, requirió menor dosis para alcanzar la capacidad antioxidante $\left(\mathrm{CE}_{50}=0,159 \mathrm{mg} / \mathrm{mL}\right)$; mediante el método $\mathrm{ABTS}(5,834 \mathrm{uM}$ trolox/g). La Caiophora carduifolia $(0,87 \mathrm{mg} / \mathrm{mL}-0,44 \mathrm{mg} / \mathrm{mL})$ tuvo efectos similares al trolox $(p>0,05)$. El Aloe vera y Plukenetia volubilis también tuvieron capacidad antioxidante dependiente de la dosis. Conclusiones. Se ha demostrado capacidad antioxidante in vitro a concentración dependiente, siendo mayor la de Cecropia membranacea y Caiophora carduifolia y menor la de Aloe vera y Plukenetia volubilis.
\end{abstract}

Palabras clave. Antioxidante, DPPH, ABTS, Aloe vera, Plukenetia volubilis, Caiophora carduifolia, Cecropia membranacea.

\section{Abstract}

Introduction. Antioxidants have shown chemopreventive potential in degenerative, inflammatory, autoimmune, oncology and respiratory distress associated pathologies. Objective. To assess the phytochemical and antioxidant properties in vitro by DPPH and ABTS method. Design. Observational analytical. Location. Laboratory of Experimental Pharmacology, Faculty of Medicine, Universidad Nacional Mayor de San Marcos, Lima, Peru. Biological/Chemical Material. Aloe vera leaves, Plukenetia volubilis seeds, Caiophora carduifolia leaves-stalks, Cecropia membranacea leaves. Interventions. Observation and analysis of the antioxidant capacity by DPPH-method mean effective concentration $\left(\mathrm{EC}_{50}\right)$ of the extracts and trolox equivalent antioxidant capacity by the ABTS method. Main outcome measures. Phytochemical preliminary march, antioxidant percent uptake inhibition by DPPH radical, determination of equivalent trolox $/ g_{\text {extract }}$ Results. Cecropia membranacea presented more secondary metabolites, alkaloids, saponins, flavonoid compounds; required smaller dosage to achieve the antioxidant effect $\left(E_{50}=0.159 \mathrm{mg} / \mathrm{mL}\right)$ in attracting DPPH radical; achieved the best antioxidant effect by ABTS method (5.834 uM trolox/g). Caiophora carduifolia $(0.87 \mathrm{mg} / \mathrm{mL}-0.44 \mathrm{mg} / \mathrm{mL}$ ) had trolox similar effects ( $p>0.05)$. Aloe vera and Plukenetia volubilis also had antioxidant dose dependent effect. Conclusions. Concentration dependent in vitro antioxidant effect has been shown, higher with Cecropia membranacea and Caiophora carduifolia and lower with Aloe vera and Plukenetia volubilis.

Keywords. Antioxidant effect, DPPH, ABTS, Aloe vera, Plukenetia volubilis, Caiophora carduifolia, Cecropia membranacea.

An Fac med. 2016;77(1):9-13 / http://dx.doi.org/10.15381/anales.v77i1.11546 


\section{INTRODUCCIÓN}

Las plantas poseen taninos, lignanos, estilbenos, compuestos fenólicos, flavonoides, catequinas, antocianinas ${ }^{(1)}$. Estos productos de origen natural demostraron potencial quimiopreventivo en bioensayos y modelos animales, los cuales han sido asociados a la actividad antioxidante ${ }^{(2)}$; como suplementos dietéticos protegen los efectos nocivos de la oxidación y neutralizan los efectos adversos del estrés oxidativo ${ }^{(3)}$. El desbalance entre oxidantes-antioxidantes está asociado a diversas patologías, como ateroesclerosis, cáncer, enfermedad de Alzheimer, diabetes mellitus, enfermedades autoinmunes, lesiones inflamatorias crónicas, situaciones de injuria por isquemia, síndrome de distrés respiratorio ${ }^{(4)}$. Los antioxidantes primarios neutralizan los radicales libres, mediante transferencia de un átomo de hidrógeno o electrón; mientras que los antioxidantes secundarios neutralizan catalizadores prooxidantes ${ }^{(3)}$. El Aloe vera 'sábila' es una especie xerofita y suculenta, originaria del África; se le ha identificado 75 principios activos ${ }^{(5)}$; presenta compuestos fenólicos, principalmente cromonas y antroquinonas, ubicados en la capa interna de las células epidermales ${ }^{\left({ }^{6}\right)}$; el parénquima gelatinoso e incoloro está constituido principalmente por agua, mucilagos, ácidos y sales orgánicas, enzimas, saponinas, taninos, trazas de alcaloides y vitaminas ${ }^{(7)}$. Plukenetia volubilis 'sacha inchi' es una especie que se encuentra en la selva tropical peruana; es reconocida por el alto contenido de proteínas, tocoferoles, aceites ricos en omega-3 y omega-6 ${ }^{(8)}$. Mediante HPLC se cuantificó compuestos fenólicos -ácidos fenólicos, flavonoides y fitoesteroles-, encontrándose estigmasterol 75,49 mg/100 g y $\beta$-sitosterol $74,62 \mathrm{mg} / 100 \mathrm{~g}{ }^{(9)}$. Caiophora carduifolia 'ortiga colorada' pertenece a la familia Loasaceae, crece en los Andes a 4000 msnm, desde Argentina, Chile, Perú hasta Ecuador ${ }^{(10)}$; es utilizada para el tratamiento del resfriado común, tos, infecciones bronquiales, neumonía y como antiinflamatorio. En un estudio fitoquímico se identificó la presencia de taninos, flavonoides, aminoácidos y alcaloides ${ }^{(11)}$. Cecropia membranacea 'guarumo' es una especie originaria de América tropical, crece a nivel del mar hasta los $1500 \mathrm{msnm}$, pertenece a la familia Urticaceae, las hojas de la planta poseen azúcares, se reportan flavonoides y esteroides ${ }^{(12)}$.

El objetivo del estudio fue evaluar las características fitoquímicas y la capacidad antioxidante in vitro de Aloe vera, Plukenetia volubilis, Caiophora carduifolia, Cecropia membranacea mediante el método DPPH y ABTS; se buscó reducir radicales libres y con ello proteger al organismo de los daños causados por el estrés oxidativo; además de generar estudios sobre los recursos vegetales del Perú.

\section{MÉTODOS}

Las muestras fueron recolectadas de diversas ciudades del Perú e identificadas por el Museo de Historia Natural de la Universidad Nacional Mayor de San Marcos (UNMSM): Aloe vera (L.) Burman f., ciudad de Lima a $160 \mathrm{msnm}$ (N¹83-USM-2015); Plukenetia volubilis Linnaeus, ciudad de Tarapoto a 330 msnm ( $\left.\mathrm{N}^{\circ} 348-\mathrm{USM}-2014\right)$; Caiophora carduifolia C. Presl, ciudad de Huari a $4000 \mathrm{msnm}$ ( $\left.\mathrm{N}^{\circ} 114-\mathrm{USM}-2015\right)$; Cecropia membranacea Trécul, ciudad de Iquitos a 104 msnm ( ${ }^{\circ} 146$ USM-2015).

Las muestras fueron procesadas a temperatura ambiente en el Laboratorio de Farmacología Experimental de la Facultad de Medicina Humana de la UNMSM; el Aloe vera fue liofilizado mediante desecación a partir de un extracto acuoso de hojas completas (500 g), colectado y procesado según Ramachandra y Srinivasa (7), posteriormente homogenizado utilizando una licuadora a $2000 \mathrm{rpm}$ durante 90 segundos; los desechos fueron separados por filtración con papel Whatman $\mathrm{N}^{\circ} 2$ para ser liofilizado y almacenado $\left(5^{\circ} \mathrm{C}\right)$; el aceite de Plukenetia volubilis fue obtenido por prensado de $900 \mathrm{~g}$ de las semillas sin cáscara a 2000 psi mediante el método de extracción del IIAP y posterior filtrado; el extracto etanólico de hojas de Cecropia membranacea $(650 \mathrm{~g})$ resultó de macerados en etanol $96^{\circ}$; el extracto hidroalcohólico de hojas-tallos de Caiophora carduifolia (500 g), por maceración en agua-etanol $96^{\circ}(1: 3)$, previa desecación y pulverizado mecánico. El liofilizado se realizó en los Laboratorios Médico Biológicos H. Colichón SAC; el prensado, en las instalaciones del Laboratorio de Farmacotecnia de la Facultad de Farmacia y Bioquímica de la UNMSM.

En relación al estudio fitoquímico preliminar según Lock (1994), las reacciones de identificación de metabolitos secundarios presentes fueron realizadas con $5 \mathrm{~mL}$ de solución y 5 gotas de reactivo ${ }^{(13)}$.

La determinación de la capacidad antioxidante in vitro se realizó en las instalaciones del Laboratorio de Farmacología Experimental de la Facultad de Medicina Humana de la UNMSM, por el método del 1,1-difenil-2-picrilhidrazilo hidratado (DPPH; SigmaAldrich $\left.{ }^{\circledR}\right)$, según Brand-Williams y col. ${ }^{(14)}$; se preparó una solución de 0,1 mM (3,9 mg DPPH + $100 \mathrm{~mL}$ de etanol), protegida de la luz; se calibró a $517 \mathrm{~nm}(0,6$ a 07) con espectrofotómetro UNICO serie 2100 .

Para cada extracto se preparó la solución madre y 2 diluciones consecutivas $(1 \mathrm{~mL}+1 \mathrm{~mL}$ de agua destilada); luego se mezcló 200 uL de solución + 1800 uL de DPPH; 30 minutos después se realizó las lecturas. Se evaluó el cambio de color del DPPH, de color violeta a un color amarillo, en presencia de antioxidantes.

La determinación de la capacidad antioxidante in vitro se realizó por el método del 2,2-azinobis-3-etilbenzotiazolin-6-sulfónico (ABTS; SigmaAldrich $($ ), según Hazra y col. (15). Se preparó el reactivo ABTS a $1 \mathrm{mM}$ (ABTS+ persulfato de potasio), 16 horas previas protegido de la luz. Para cada extracto se preparó la solución madre y 2 diluciones consecutivas (1 
$\mathrm{mL}+1 \mathrm{~mL}$ de agua destilada), luego se mezcló 1960 uL de ABTS + 40 uL de extracto; 7 minutos después se analizó la absorbancia a $734 \mathrm{~nm}$ con espectrofotómetro UNICO serie 2100.

Se utilizó el reactivo estándar TRO. LOX $1 \mathrm{mM}$ (250 ug/mL) como patrón de referencia antioxidante ${ }^{(14,15)}$.

La descripción de variables se expresa en medias, desviación estándar, porcentaje de inhibición; la comparación estadística de grupos mediante la prueba de Kruskal-Wallis; la comparación de las medias en relación al grupo control por la prueba U-Mann Whitney. Se consideró significativo un valor $\mathrm{p}<0,05$, intervalo de confianza al $95 \%$; la $\mathrm{EC}_{50}$ se determinó mediante regresión lineal.

\section{RESULTADOS}

Al realizar el análisis fitoquímico, se identificó la mayor presencia de metabolitos secundarios en Cecropia membranacea, siendo la única en presentar alcaloides; además, junto con la Caiophora carduifolia presentaron aminoácidos libres y saponinas; todas las plantas presentaron compuestos fenólicos y flavonoides, a excepción de Plukenetia volubilis, que solo mostró triterpenos y esteroides; asimismo, el Aloe vera presentó antronas y naftoquinonas (tabla 1).

En la evaluación de captación de radical DPPH, el porcentaje de inhibición de la capacidad antioxidante se observó en un efecto dosis-respuesta; el análisis de diferencia de medias Kruskal-Wallis mostró diferencia significativa $(\mathrm{p}<0,05)$ entre los grupos. El grupo Plukenetia volubilis $(1,06 \mathrm{mg} / \mathrm{mL})$, Caiophora carduifolia $(0,87 \mathrm{mg} / \mathrm{mL}, 0,44 \mathrm{mg} /$ $\mathrm{mL}$ ) lograron un resultado similar al trolox (U-Mann Whitney $(\mathrm{p}>0,05)$ ); Cepropia membranacea $(0,07 \mathrm{mg} / \mathrm{mL})$, Caiophora carduifolia $(0,22 \mathrm{mg} / \mathrm{mL})$ presentaron menor inhibición en relación al trolox (U-Mann Whitney $(\mathrm{p}<0,05))$ (tabla 2). Asimismo, todas mostraron capacidad antioxidante superior al $25 \%$, excepto Aloe vera $(0,30 \mathrm{mg} / \mathrm{mL})$ (figura 1).
Tabla 1. Marcha fitoquímica.

\begin{tabular}{cccccc} 
Metabolito secundario & Reacción & $\begin{array}{c}\text { Aloe } \\
\text { vera }\end{array}$ & $\begin{array}{c}\text { Plukenetia } \\
\text { Volubilis }\end{array}$ & $\begin{array}{c}\text { Caiophora } \\
\text { carduifolia }\end{array}$ & $\begin{array}{c}\text { Cecropia } \\
\text { Alcaloides }\end{array}$ \\
Taninos & Mayer & - & - & - & + \\
AA libres & Gelatina & - & - & - & - \\
Flavonoides & Agua de Br & - & - & + & + \\
Comp. fenólicos & Shinoda & + & - & + & + \\
Saponinas & Cloruro férrico & + & - & + & + \\
Triterpenos y/o esteroides & Ensayo de espuma & - & - & + & + \\
Antronas y naftoquinonas & Bobermann-Burchard & - & + & - & + \\
\hline
\end{tabular}

(+) Presencia, (-) Ausencia.

Tabla 2. Efecto antioxidante in vitro mediante el método DPPH.

\begin{tabular}{|c|c|c|c|}
\hline \multirow{2}{*}{$\begin{array}{l}\text { Grupo experimental } \\
\qquad(\mathrm{N}=6)^{* *}\end{array}$} & \multicolumn{3}{|c|}{ Porcentaje de inhibición DPPH $(\%)^{*}$} \\
\hline & $1^{\circ}$ Dilución ${ }^{* * *}$ & $2^{\circ}$ Dilución ${ }^{* * *}$ & $3^{\circ}$ Dilución ${ }^{* * *}$ \\
\hline $\begin{array}{c}\text { Trolox } \\
\text { (250ug/mL }-125 u g / m L-62,5 u g / m L)\end{array}$ & $94,66 \pm 0,04$ & $53,19 \pm 0,19$ & $26,52 \pm 0,34$ \\
\hline $\begin{array}{c}\text { Aloe vera } \\
(1,21 \mathrm{mg} / \mathrm{mL}-0,61 \mathrm{mg} / \mathrm{mL}-0,30 \mathrm{mg} / \mathrm{mL})\end{array}$ & $66,28 \pm 0,48^{b}$ & $39,19 \pm 1,91^{b}$ & $24,60 \pm 0,67^{b}$ \\
\hline $\begin{array}{c}\text { Plukenetia volubilis } \\
(4,23 \mathrm{mg} / \mathrm{mL}-2,11 \mathrm{mg} / \mathrm{mL}-1,06 \mathrm{mg} / \mathrm{mL})\end{array}$ & $76,88 \pm 0,25^{b}$ & $46,34 \pm 2,42^{b}$ & $26,52 \pm 0,09^{a}$ \\
\hline $\begin{array}{c}\text { Caiophora carduifolia } \\
(0,87 \mathrm{mg} / \mathrm{mL}-0,44 \mathrm{mg} / \mathrm{mL}-0,22 \mathrm{mg} / \mathrm{mL})\end{array}$ & $94,42 \pm 0,78^{a}$ & $53,29 \pm 0,48^{a}$ & $35,32 \pm 1,44^{b}$ \\
\hline $\begin{array}{c}\text { Cecropia membranacea } \\
(0,29 \mathrm{mg} / \mathrm{mL}-0,15 \mathrm{mg} / \mathrm{mL}-0,07 \mathrm{mg} / \mathrm{mL})\end{array}$ & $80,63 \pm 1,01^{b}$ & $48,68 \pm 0,00^{\mathrm{b}}$ & $30,74 \pm 0,10^{b}$ \\
\hline
\end{tabular}

*Valores expresados en media \pm desviación estándar. ${ }^{* *}$ Kruskal Wallis $(P=0.01) .{ }^{* * *}$ Variaciones de la inhibición de las soluciones experimentales en relación al trolox. U-Mann Whitney a $(p>0,05), b(p<0,05)$.

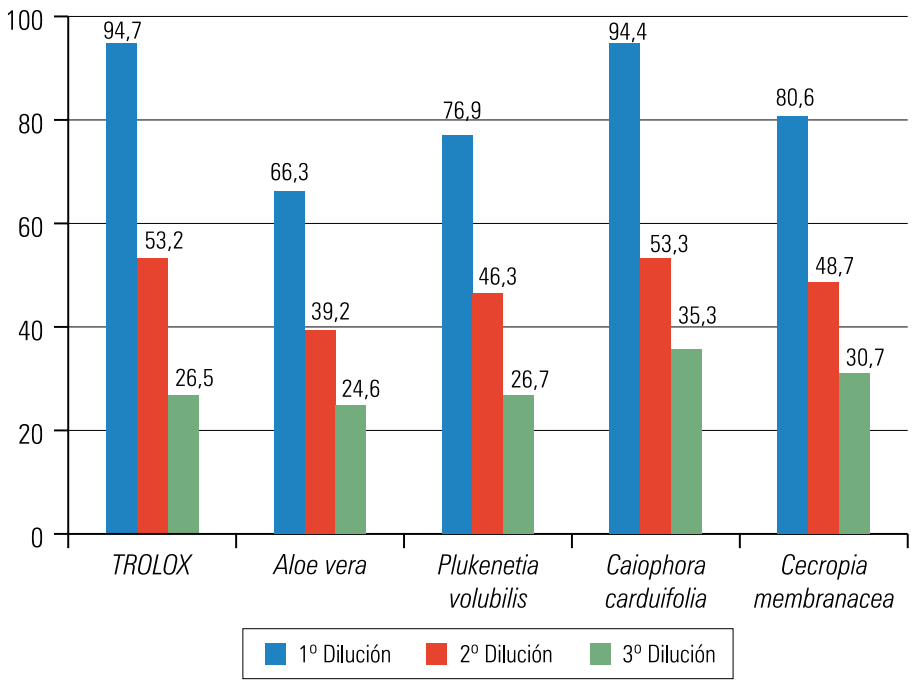

Figura 1. Porcentaje de Inhibición del trolox y extractos mediante el método DPPH. 
Tabla 3. Efecto antioxidante in vitro mediante el método DPPH y ABTS.

\begin{tabular}{cccc} 
& DPPH & & ABTS $^{*}$ \\
\cline { 2 - 3 } Aloe vera & $\mathrm{EC}_{50}(\mathrm{mg} / \mathrm{mL})$ & & $\mathrm{uM}$ trolox/g extracto \\
Plukenetia volubilis & $0,860 \pm 0,01$ & & $1,160 \pm 0,060$ \\
Caiophora carduifolia & $2,550 \pm 0,04$ & & $0,154 \pm 0,001$ \\
Cecropia membranacea & $0,420 \pm 0,06$ & & $4,580 \pm 0,020$ \\
\hline
\end{tabular}

EC50: Concentración efectiva media. *Desviación estándar.

A partir del porcentaje de inhibición-dosis en el DPPH y mediante un análisis lineal se determinó la $\mathrm{EC}_{50}$ (tabla 3); demostrando que la Cecropia membranacea requirió menor cantidad para alcanzar una actividad antioxidante $(0,159 \mathrm{mg} / \mathrm{mL})$, seguido de la Caiophora carduifolia $(0,420 \mathrm{mg} / \mathrm{mL})$ y Aloe vera $(0,860 \mathrm{mg} / \mathrm{mL})$. Asimismo, mediante el método del ABTS se observó mayor capacidad antioxidante del extracto de Cecropia membranacea y Caiophora carduifolia (tabla 3).

\section{DISCUSIÓN}

En el presente estudio se evidenció la presencia de diversos metabolitos secundarios que confieren capacidad antioxidante; los compuestos fenólicos presentes en Aloe vera, Caiophora carduifolia y Cepropia membranacea actúan como interruptores de radicales libres o quelantes de metales, provocando un efecto antioxidante ${ }^{(16)}$.

La capacidad antioxidante ha sido evidente con Cepropia membranacea, posiblemente porque tiene una mayor cantidad de metabolitos secundarios (tabla 1); con resultados similares encontrados por Ospina y col., la Cecropia peltata posee flavonoides, taninos y menor proporción de terpenos y esteroides; además demostró poseer un efecto neurofarmacológico ${ }^{(17)}$ y se encontró alcaloides en los géneros Cecropia polyphlebia y Cecropia obtusifolia ${ }^{(18)}$; esto coincidió con lo hallado en la marcha fitoquímica.

Estudios realizados de Caiophora sepiaria y Caiophora ahmeri indicaron que no poseen capacidad antioxidan- te ${ }^{(10)}$. Sin embargo, Khera y col. ${ }^{(19)}$ identificaron triterpenos, en la especie Caiophora coronata; además, observando efectos antibacterianos, estos datos coinciden con los resultados obtenidos en la marcha fotoquímica. Se presume que la presencia de saponinas, flavonoides y aminoácidos libres contribuyen a alcanzar altos potenciales de inhibición mediante el método de DPPH y ABTS ${ }^{(20)}$; con ello contribuyen a la capacidad antioxidante in vitro.

Del mismo modo, el aceite de Plukenetia volubilis logró un elevado valor de $\mathrm{EC}_{50}$ con baja capacidad antioxidante equivalente al trolox; se puede atribuir ello a la ausencia de compuestos fenólicos y flavonoides, principales metabolitos secundarios responsables de la capacidad antioxidante ${ }^{(21)}$, los cuales se encuentran principalmente en las hojas de la planta según el reporte de Castillo y col. ${ }^{(22)}$.

Rahda y col. y Lucini y col. refieren alto contenido de compuestos flavonoides y antroquinonas del Aloe vera ${ }^{(5,6)}$; la presencia de estos metabolitos secundarios coinciden con los resultados obtenidos en la marcha fitoquímica. Los anillos de flavonoides poseen un gran potencial para inhibir la generación de especies reactivas de oxígeno; los flavonoides glicosilados se encuentran con frecuencia en hojas sanas y poseen actividad reductora ${ }^{(23)}$.

La presencia de antroquinonas en Aloe vera y Cecropia membranacea podría contribuir en la capacidad antioxidante mediante la peroxidación de lípidos; adicionalmente, se le atribuye propiedades hepatoprotectoras, inmunomoduladoras e inhibidores de canales de calcio ${ }^{(24)}$.
De todos los metabolitos secundarios, los compuestos fenólicos muestran una correlación lineal entre la concentración y la capacidad antioxidante expresada en equivalentes de trolox y ácido ascórbico ${ }^{(25)}$; en especial los flavonoides ${ }^{(26)} \mathrm{y}$ asimismo los fitoesteroles como el $\beta$-sitosterol poseen también dicho efecto leve o moderado in vitro, reduciendo el estrés oxidativo causado por la administración de 1,2-dimetilhidracina durante la inducción de carcinogénesis en colon de ratas (27).

Se concluye que las plantas del estudio poseen metabolitos secundarios, principalmente compuestos fenólicos y flavonoides; además, se observa mayor capacidad antioxidante in vitro de Cecropia membranacea y Caiophora carduifolia por el método DPPH y ABTS y menor capacidad antioxidante para Aloe vera y Plukenetia volubilis, siendo el efecto dependiente de la concentración.

\section{AGRADECIMIENTOS}

Al Q.F. Miguel Abregu Flores, jefe de producción en Laboratorios Médico Biológicos H. Colichón SAC por brindarnos las facilidades para el procesamiento de las muestras.

Al laboratorio de Farmacología Experimental de la Facultad de Medicina Humana de la UNMSM por brindarnos sus instalaciones para la realización de la presente investigación.

\section{REFERENCIAS BIBLIOGRÁFICAS}

1. Marwah R, Fatope M, Mahrooqi R, Varma G,Abadi $\mathrm{H}$, Khamis S, et al. Antioxidant capacity of some edible and wound healing plants in Oman. Food chemistry. 2007;101:465-70.

2. Chia-Jui W, Gow-Chin Y. Natural plant extracts as antioxidants for food preservation. A volume in Woodhead. UK:Technology and Nutrition. 2015:235-49.

3. Embuscado M. Handbook of antioxidants for food preservation. A volume in Woodhead Publishing Series in Food Science. UK: Technology and Nutrition. 2015:251-83.

4. Mayor R. Estrés oxidativo y sistema de defensa antioxidante. R Inst Med Trop. 2010;5(2):23-39.

5. Rahda M, Laxmipriya N. Evaluation of biological properties and clinical effectiveness of Aloe vera: A systematic review. J Trad Complem Med. 
2015;5:21-6. doi: 10.1016/j.jtcme.2014.10.006

6. Lucini L, Pellizzoni M, Pellegrino R, Mollinari G, Colla $\mathrm{G}$. Phytochemical constituents and in vitro radical scavenging activity of different Aloe species. Food Chemistry. 2015;170:501-7. doi: 10.1016/j. foodchem.2014.08.034.

7. Dominguez R, Arzate I, Chanona J, Welti J, Alvarado J, Calderón $\mathrm{G}$, et al. El gel de Aloe vera: estructura, composición química, procesamiento, actividad biológica e importancia en la industria farmacéutica y alimentaria. Rev Mex Ingeniería Quim. 2012;11(1):23-43.

8. Wang X, Xu R, Wang R, Liu A. Transcriptome analysis of Sacha Inchi (Plukenetia volubilis L.) seeds at two developmental stages. BMC Genomics. 2012 Dec 20;13:716. doi: 10.1186/1471-2164-13-716.

9. Muñoz A, Ramos F, Ortiz C, Castañeda B, Barnett E, Yáñez J y col. Evaluación del contenido de fitoesteroles, compuestos fenólicos y métodos quimicos para determinar la actividad antioxidante en semilla de sacha inchi (Plukenetia volubilis L.). Rev Soc Quim Perú. 2010;76(3):234-41.

10. Matta M. Screening fitoquímica, antimicrobiano y antioxidante de plantas pre-andinas y del altiplano chileno (Tesis para optar al título de Químico Farmacéutico). Chile. Univ de Chile. 2009.

11. Puelles M, Gómez V, Gabriel J, Moris G. Las plantas medicinales de perú: etnobotánica y viabilidad comercial. Madrid. 2010:43 y 130.

12. Hernández J, Luengas $P$. Estudio fitoquímico preliminar de Cecropia membranacea trecul. Y Cecropia metensis Cuatrec. Rev Cubana Plantas Med. 2013;18(4):586-95.

13. Lock O. Investigación fitoquímica. Métodos en el estudio de productos naturales. 2da edición. Lima: Fondo Editorial Pontificia Universidad Católica del Perú. 1994:33-5.

14. Brand-Williams W, Cuvelier M, Berset C. Use of free radical method to evaluate antioxidant activity. Lebensmittel Wissenschaftund Technologie. 1995;28:25-30.
15. Hazra B, Biswas S, Mandal N. Antioxidant and free radical scavenging activity of Spondia spinnata BMC Complement Altern Med. 2008;8:63.

16. Lee KY, Weintraud ST, Yu BP. Isolation and identification of phenolic antioxidant from Aloe barbadensis. Free Radic Biol Med. 2000;28(2):261-5.

17. Ospina J, Rincón J, Guerrero M. Perfil neurofarmarmacológico de la fracción butanólica de las hojas de Cecropia peltata. Rev Colomb Cienc Quim Farm. 2013;42(2):244-59.

18. Xiong M. The effects of Cecropia obtusifolia and Cecropia polyphlebia on lung capacity in relation to alkaloid concentration. University of South Florida. 2009

19. Khera S, Woldemichael G, Singh M, Suarez E, Timmermann B. A novel antibacterial iridoic and triterpene from Caiophora coronate. J Natural Products. 2003;66(12):1628-31.

20. Raghavendra M, Madhusudhana A, Raghuveer $P$, Sudharshan A, Siva I. Comparative studies on the in vitro antioxidant properties of methanolic leafy extracts from six edible leafy vegetables of india. Asian J Pharm Clin Res. 2013;6(3):96-9.

21. Arroyo J, Raez E, Rodríguez M, Chumpitaz V Burga J, De la Cruz W. Reducción del colestero y aumento de la capacidad antioxidante por el consumo crónico de maíz morado (Zea mays L) en ratas hipercolesterolémicas. Rev Perú Med Exp Salud Publica. 2007;24(2):157-62.

22. Castillo E, Castillo S, Reyes C. Estudio fitoquimico de Plukenetia volubilis I. y su efecto antioxidante en la lipoperoxidación inducida por $\mathrm{fe}^{3+} / \mathrm{ascorb}$ ato en higado de Rattus rattus var. Albinus. UCV Scientia. 2010;2(1):11-21.

23. Agati G, Azzarello E, Pollastri S, Tattinin M. Flavonoids as antioxidant in plants: location and functional significance. Plant Science. 2012;196:67-76.

24. Jeevitha D, Amarnath K. Chitosan/PLA nanoparticles as a novel carrier for the delivery of anthraquinone: Synthesis, characterization and in vitro cytotoxicity evaluation. Colloids and Surfaces B: Biointerfaces. 2013;101:126-34. doi: 10.1016/j. colsurfb.2012.06.019
25. Velásquez H, Garcia P. Evaluación fitoquímica y de actividad antioxidante de los rizomas de tres especies del género Cyperus. Momentos de Ciencia. 2012;9(1):15-21.

26. Sumczynski D, Bubelova Z, Sneyd J, ErbWeber S, Icek, J. Total phenolics, flavonoids, antioxidant activity, crude fibre and digestibility in non-traditional wheatflakes and muesli. Food chemistry. 2015;174:319-25. doi: 10.1016/j.foodchem.2014.11.065

27. Saeidnia S, Manayi A, Gohari A, Abdollahi M. The story of beta-sitosterol-a review. Eur J Med Plants. 2014;4(5):590-609.

Artículo recibido el 11 de agosto de 2015 y aceptado para publicación el 6 de noviembre de 2015.

El presente articulo se presenta de manera exclusiva, no se envió a otra revista científica.

Financiamiento: El costo del presente artículo fue asumido en su totalidad por los autores y colaboradores.

Conflictos de interés: El presente artículo no presenta conflicto de intereses.

\section{Correspondencia:}

César Ivanovish Franco Quino

Dirección: Laboratorio de Fisiología y Farmacología,

Facultad de Odontologia, UNMSM, Ciudad

Universitaria, Lima, Perú.

Telefono: 996517008

Correo electrónico: cesar.franco1@unmsm.edu.pe c.francoq@hotmail.com 